\title{
The importance of body mass normalisation for ultrasound measurements of the morphology of oblique abdominis muscles: the effect of age, gender, and sport practice
}

\author{
P. Linek \\ Department of Kinesitherapy and Special Methods in Physiotherapy, Jerzy Kukuczka Academy of Physical Education, \\ Katowice, Poland
}

[Received: 18 May 2017; Accepted: 23 May 2017]

Some studies have not considered body mass as a confounder in analysis of oblique abdominis muscles (OAM) (including the oblique externus [OE] and oblique internus $[O I]$, which may have led to improper interpretation of results. To assess the differences in the effect of age, gender, and physical activity between normalised for body mass and actual values of the OAM as well as to establish the effect of age, gender, and physical activity on normalised for body mass OAM thicknesses in adolescents. A real-time ultrasound was used to obtain images of the OAM. Body mass normalisation for OAM thicknesses was performed with allometric scaling and the following equations: Allometric-scaled $O E=O E$ thickness/body mass ${ }^{0.88}$; Allometric-scaled $\mathrm{OI}=\mathrm{O}$ I thickness/body mass ${ }^{0.72}$. Analysis showed that boys have significantly thicker OAM than girls, and those who practise sports have thicker OAM than non-active individuals. For allometric-scaled OAM, there was only a significant gender effect, where boys have thicker allometric-scaled OAM than girls. There was a significant correlation between participants' age and the actual value of the OAM. The correlations between age and allometric-scaled OAM were insignificant. An analysis of OAM without body mass normalisation can lead to improper interpretation of study results. Thus, future studies should analyse OE and OI thickness measurements after normalisation rather than actual values. In the adolescent population, there is no effect of age and physical activity on allometric-scaled OAM; males have thicker allometric-scaled OAM than females. (Folia Morphol 2018; 77, 1: 123-130)

Key words: body mass, normalisation, allometric scaling, oblique internus, oblique externus, abdominal wall morphology

\section{INTRODUCTION}

The lateral abdominal wall is constituted of three different muscles: the oblique externus (OE), oblique internus (OI), and transversus abdominis (TrA). All of them have been widely examined by ultrasound imaging, which is a relatively simple and cost-effective tool allowing reliable assessment of the muscles in different populations $[12,15,28]$. Some studies have tried to find some connection between $\mathrm{OE}, \mathrm{OI}$, and TrA morphology and conditions, such as low back pain and scoliosis $[3,5,11,16,25]$. To better understand lateral abdominal wall morphology, some studies have also examined

Address for correspondence: Dr. P. Linek, Department of Kinesitherapy and Special Methods in Physiotherapy, Jerzy Kukuczka Academy of Physical Education, ul. Mikołowska 72B, 40-065 Katowice, Poland, e-mail: linek.fizjoterapia@vp.pl 
the effect of age, gender, physical activity, as well as anthropometric measurements on the $\mathrm{OE}, \mathrm{OI}$, and $\operatorname{Tr} \mathrm{A}$ thickness in adult and adolescent healthy populations $[13,17,18,22,27]$. These works have shown that body mass is highly correlated with all lateral abdominal muscle thicknesses $[14,17,18,22]$. Thus, it seems necessary to take into account body mass as a confounder in the research on these muscle morphologies [14].

Recently, it has been shown that analysis of TrA morphology without body mass normalisation can lead to improper interpretation of study results in the adolescent population [10]. Linek [10] confirmed that prior to body mass normalisation, there is an effect of age, gender, and physical activity on TrA thickness, whereas in reality (after normalisation) there is no age, gender, or physical activity effect on normalised TrA thickness. Thus, it was recommended to analyse the TrA thickness measurement after normalisation rather than the actual value in future studies [10]. During ultrasound imaging measurement of the TrA morphology, the morphology of the OE and $\mathrm{OI}$ is usually also assessed. Thus, it is reasonable to address the problem of possible misinterpretation of the $\mathrm{OE}$ and $\mathrm{OI}$ thickness measurements in scientific research.

In some papers, the oblique abdominis muscles (OAM) morphology was analysed without consideration of body mass $[9,17,18,23,24]$, which may have led to inaccurate interpretation of the results. As an example, females demonstrate lower resting thickness of the $\mathrm{OE}$ and $\mathrm{OI}$ compared to males, but females also have a significantly lower body mass value $[17,22$, 23]. The same problem of possible misinterpretation can be seen in studies evaluating the effect of physical (sport) activity and/or age on OAM thicknesses $[9,18$, $22,26]$. Thus, the effect of gender, age, and physical activity on $\mathrm{OE}$ and $\mathrm{OI}$ thicknesses may be caused only by body mass, as was seen for TrA [10].

Nuzzo and Mayer [21] examined two ways of body mass normalisation and confirmed that allometric scaling is better than ratio scaling for the $\mathrm{OE}, \mathrm{OI}$, and TrA. The third popular normalisation method involves analysis of covariance (ANCOVA), in which body mass could be introduced as a covariate in a statistical model. However, the ANCOVA analysis requires multiple assumptions, which have to be met to properly perform such a statistical procedure. Thus, scaling procedures seem to be a more practical and easier way to normalise ultrasound imaging thickness of the $\mathrm{OE}, \mathrm{OI}$, and TrA to body mass. The preferable allometric scaling procedure is based on the theory of geometrical symmetry suggesting that all humans have the same shape but are of different sizes [8]. In practice, the allometric method requires a constant value of the allometric parameter (the so-called exponential power), which is then used to normalise a given muscle thickness ultrasound imaging measurement to body mass [8]. To date, the allometric parameter for the $\mathrm{OE}, \mathrm{OI}$, and $\operatorname{Tr} \mathrm{A}$ has been assessed only in a healthy adolescent population; the value was $0.88,0.72$, and 0.61 , respectively [14].

The confounding effect of body mass on OAM morphology is important in scientific research on different populations, but since weight is physiologically doubled in adolescence [2], $\mathrm{OE}$ and $\mathrm{OI}$ muscle normalisation is of greater importance in adolescent populations. The present study is the first to address the problem of misinterpretation of the results of OAM thicknesses analysis in an adolescent population due to differences in body mass. Potential confounders such as age, gender, and sport practice were used as an example to highlight the problem. The aims of the study were: 1 ) to assess differences in the effects of age, gender, and physical activity between values normalised for body mass and the actual value of the $\mathrm{OE}$ and $\mathrm{OI}$, and 2) to establish the effect of age, gender, and physical activity on normalised for body mass OE and OI thickness (the effect independent of body mass).

\section{MATERIALS AND METHODS}

\section{Setting and study design}

This was a cross-sectional study conducted at selected primary and secondary schools and colleges in the Silesian region of Poland. It was designed in accordance with the Declaration of Helsinki and was approved by the local medical ethics committee. All participants and their parents and/or legal guardians received oral and written information about all procedures and gave their signed informed consent to participate.

\section{Study population}

The population invited for the study was from local schools and colleges (age span 10-17 years). All of them attended regular school classes at normal grade levels. At the beginning of the study, participants answered questions related to their medical history, and information from the child's health record at school was analysed. The following individuals were excluded: 1) those in whom any surgical procedure had been performed on the pectoral chest, abdominal cavity, pelvic girdle, and/ or spine; 2 ) those with a chronic cardiovascular/respira- 
tory system disorder; and 3) those who had experienced pain in the spine, pelvic girdle, and/or lower limbs during the three months preceding the study.

For included participants, a traditional mechanical physician's medical body weight scale was used to assess body mass. Immediately after body mass assessment, body rotation (axial trunk rotation [ATR]) was tested with a scoliometer in a forward bending position (Adams test). This is a common procedure used in screening for scoliosis in the adolescent population [4]. During body weight and ATR measurements, boys were evaluated shirtless, and girls wore a swimsuit that allowed the entire back to be seen. Female participants also had their hair tied up. All participants were barefoot.

Finally, each subject responded to questions about current and past physical activities. This allowed division of the participants into two groups: active and inactive. Participants who did do not practise any sports were included in the non-physically active group (Sport - no), whereas participants who stated that they had belonged to a sport club for at least one year, and therefore regularly practised a specific sport, were included in the physically active group (Sport - yes). Detailed information on examined groups is presented in Table 1.

\section{Ultrasound scanning procedure}

A real-time ultrasound B-scanner (Honda HS 2100, Honda Electronics Co. Ltd., Medical Division, Toyohashi, Japan) with a linear array transducer (HLS-575M) was used to obtain images of the OE and OI. The transducer was always placed on the anterolateral wall of the abdomen between the iliac crest and the costal margin, perpendicular to the longitudinal axis of the body, and was finally adjusted to ensure that, at rest, the fascial borders of the OE and OI appeared parallel on the screen.

Measurements of the thickness of the OE and OI at rest were made in the supine rest position and from both body sides, separately. During the ultrasound imaging procedure, the knees of the examined individuals were extended and the upper limbs placed along the sides of the trunk [12-14, 17]. The thicknesses of the muscles were recorded at the end of normal expiration. All images were saved on an external drive in jpg format.

In order to increase the precision of the measurements, all images were transferred to a computer, where they were further processed using Photoshop software (Adobe Systems, Inc. San Jose, CA, USA) (Fig. 1), which has been used previously for the evaluation of the thickness of the lateral abdominal muscles in adolescents
Table 1. Demographic characteristics of the subjects*.

\begin{tabular}{|c|c|c|}
\hline \multirow[t]{2}{*}{ Parameter } & \multicolumn{2}{|c|}{ Gender } \\
\hline & $\begin{array}{c}\text { Girls } \\
(n=91)\end{array}$ & $\begin{array}{c}\text { Boys } \\
(n=91)\end{array}$ \\
\hline Age [years] & $14.2 \pm 2.44$ & $14.1 \pm 2.03$ \\
\hline Weight $[\mathrm{kg}]$ & $51.1 \pm 10.8$ & $58.7 \pm 14.6$ \\
\hline Height [cm] & $158.7 \pm 10.5$ & $166.9 \pm 14.2$ \\
\hline Sport practising - Yes [\%] & $37 \pm 40.6$ & $44 \pm 48.3$ \\
\hline Football & - & $23 \pm 52.2$ \\
\hline Volleyball & $18 \pm 48.6$ & $12 \pm 27.3$ \\
\hline Ju Jitsu & $5 \pm 13.5$ & $7 \pm 15.9$ \\
\hline Dance & $10 \pm 27.0$ & - \\
\hline Basketball & - & $1 \pm 2.3$ \\
\hline Swim ski & $1 \pm 2.7$ & $1 \pm 2.3$ \\
\hline Running & $1 \pm 2.7$ & - \\
\hline Swimming & $1 \pm 2.7$ & - \\
\hline Badminton & $1 \pm 2.7$ & - \\
\hline Sport practising [years] & $3.19 \pm 2.74$ & $3.42 \pm 1.91$ \\
\hline \multicolumn{3}{|l|}{ Axial trunk rotation [degree] } \\
\hline Thoracic (T6) & $1.05 \pm 1.6$ & $1.26 \pm 1.6$ \\
\hline $\min -\max$ & $0-8$ & $0-6$ \\
\hline Thoraco-lumbar (Th/L) & $1.55 \pm 2.0$ & $1.48 \pm 2.1$ \\
\hline $\min -\max$ & $0-10$ & $0-10$ \\
\hline Lumbar (L3) & $1.42 \pm 1.8$ & $1.70 \pm 2.0$ \\
\hline $\min -\max$ & $0-7$ & $0-10$ \\
\hline
\end{tabular}

*Values are mean \pm standard deviation unless otherwise indicated

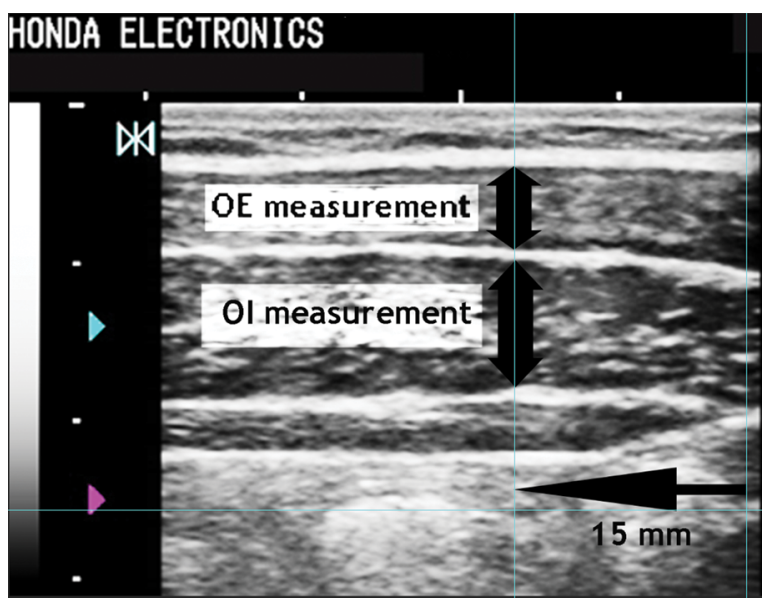

Figure 1. The method used to quantify the thickness of the oblique externus $(\mathrm{OE})$ and oblique internus $(\mathrm{OI})$ muscle. The method contained insertion of horizontal and vertical lines in the appropriate places $(15 \mathrm{~mm}$ from musculofascial junction of the transversus abdominis) and before the measurement image was enlarged $(300 \%$ - not shown here), made brighter and contrast was added. 
$[9,13,14]$. The mean of three measurements from three separate images of the $\mathrm{OE}$ and $\mathrm{OI}$ obtained from each body side was further analysed. All ultrasound imaging procedures and Photoshop measurements were performed by one investigator, who is a physiotherapist and has a few years of experience of assessing lateral abdominal muscles in adolescent and adult populations.

\section{Muscles normalisation}

The allometric parameters necessary for the scaling procedure were from a recently published paper and for the $\mathrm{OE}$ and $\mathrm{OI}$ were 0.88 and 0.72 , respectively [14]. To normalise the muscle size measurements to body mass, the following equations were used:

$$
\text { Allometric-scaled } O E=\frac{O E \text { thickness }}{\text { Body mass }}{ }^{0.88}\left[\frac{\mathrm{mm}}{\mathrm{kg}^{0.88}}\right]
$$$$
\text { Allometric-scaled OI }=\frac{\text { Ol thickness }}{\text { Body mass } 0.72}\left[\frac{\mathrm{mm}}{\mathrm{kg}^{0.72}}\right]
$$

\section{Statistical analysis}

Differences in demographic data between the control and sport groups were examined using two-way analysis of variance (ANOVA). The ultrasound imaging data assessing side-to-side differences of the $\mathrm{OE}$ and Ol were analysed with two-way ANOVA for repeated measurements, with between-subjects factors being group and gender and within factor being body side (right side vs. left side). Actual values and allometricscaled values of the $\mathrm{OE}$ and $\mathrm{OI}$ were also analysed using two-way ANOVA, with between-subjects factors being group (control vs. sport) and gender (male vs. female). When significance was found, Tukey's test for post-hoc evaluation was performed. The results are presented as a mean difference and $95 \%$ confidence interval (CI). Correlations between age and ultrasound imaging data were analysed using the Pearson correlation.

For significant post-hoc dependences, to assess effect size the mean values, standard deviations, and number of participants were used. As an index of effect size, standardised Hedges' $\mathrm{g}\left(\mathrm{H}_{\mathrm{g}}\right)$ effect size for between-subjects design as well as common language effect size $(\mathrm{CL})$ were reported $[1,20]$. The $H_{g}$ effect size was interpreted as trivial $(<0.20)$, small $(0.20-0.49)$, medium $(0.50-0.79)$, and large $(\geq 0.80)$. All statistical analyses were performed with the use of Statistica 12PL software, and $p$ values $<0.05$ were considered significant for all tests.

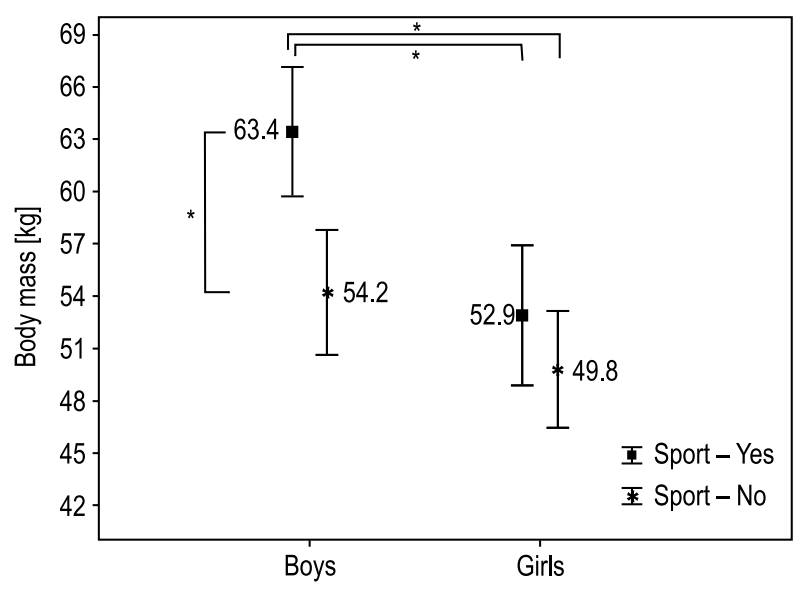

Figure 2. Body mass value in examined groups; ${ }^{*} p<0.001$.

\section{RESULTS}

\section{Participants}

For the participants' age, no statistically significant effect of gender, sport practice, and interaction (gender $\times$ sport) was observed $(p>0.05)$. Regarding body mass and body height, all effects were significant $(p<0.05)$. Compared to physically active girls and non-active boys, physically active boys were significantly heavier by $10.5 \mathrm{~kg}\left(95 \% \mathrm{Cl} 3.13-17.9, \mathrm{H}_{\mathrm{g}}=1.04\right.$, $\mathrm{CL}=77 \%)$ and $9.22 \mathrm{~kg}\left(95 \% \mathrm{Cl} 2.26-16.2, \mathrm{H}_{\mathrm{g}}=0.65\right.$, $\mathrm{CL}=68 \%$ ), respectively. Generally, the girls had a lower body mass by $7.61 \mathrm{~kg}$ than boys $(95 \% \mathrm{Cl} 3.97-$ $-11.2, \mathrm{H}_{\mathrm{g}}=0.59, \mathrm{CL}=66 \%$ ). Additionally, physically active group (boys and girls) had a higher body mass by $6.78 \mathrm{~kg}\left(95 \% \mathrm{Cl} 3.11-10.4, \mathrm{H}_{\mathrm{g}}=0.52, \mathrm{CL}=65 \%\right)$ than non-active peers (Fig. 2).

There were no significant side-to-side differences for the OI and OE $(p>0.05)$. Thus, the mean values from both sides were used in further analysis.

\section{Oblique externus}

For an actual value of the $\mathrm{OE}$, a significant main effect of sport practice, gender, and interaction was demonstrated $(p<0.05)$. A subsequent ANOVA with Tukey post hoc analysis showed that athlete boys have significantly thicker muscle by $1.11 \mathrm{~mm}(95 \% \mathrm{Cl} 0.39$ $-1.84, \mathrm{H}_{\mathrm{g}}=0.80, \mathrm{CL}=72 \%$ ) than non-athlete boys, and at least $1.39 \mathrm{~mm}\left(95 \% \mathrm{Cl} 0.62-2.16, \mathrm{H}_{\mathrm{g}}=1.10\right.$, $\mathrm{CL}=78 \%$ ) thicker than athlete and non-athlete girls (Fig. 3A). Additionally, compared to athlete participants (both genders), non-athletes have thinner $\mathrm{OE}$ muscle by $0.80 \mathrm{~mm}\left(95 \% \mathrm{Cl} 0.41-1.18, \mathrm{H}_{\mathrm{g}}=0.57, \mathrm{CL}=66 \%\right)$, 

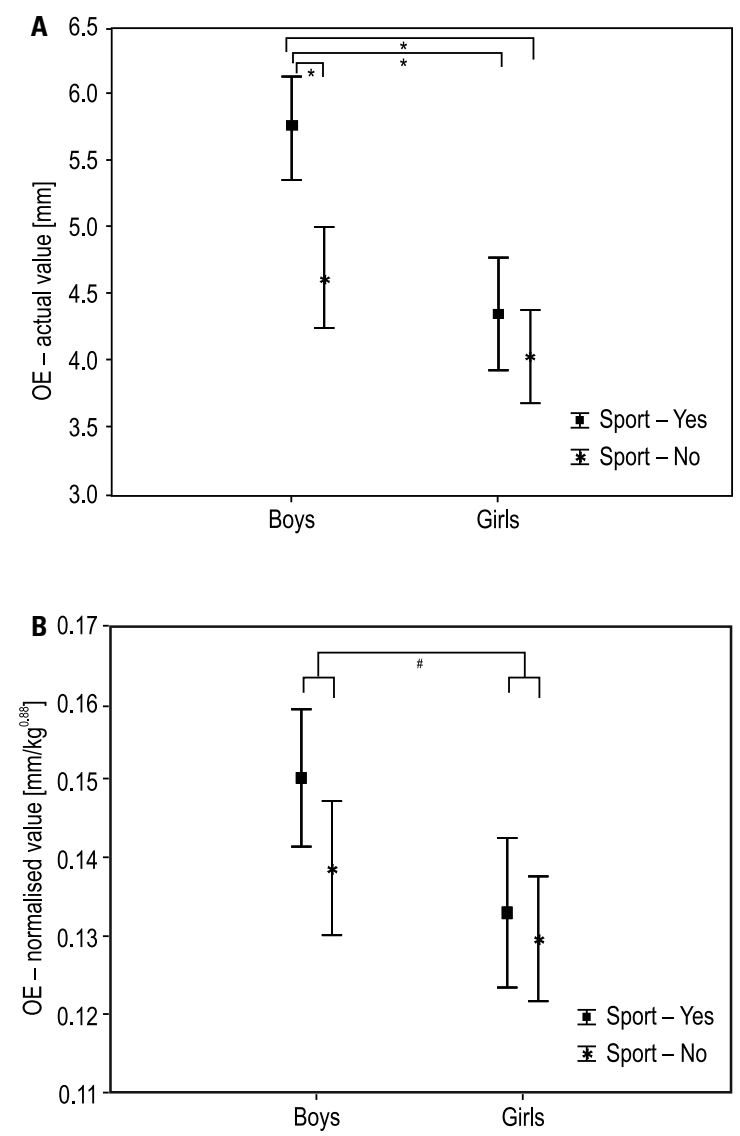

Figure 3. Oblique externus muscle $(\mathrm{OE})$ before $(\mathbf{A})$ and after (B) normalisation in examined groups; ${ }^{*} p<0.001$; ${ }^{*} p<0.003$.

and boys have thicker OE muscle by $1.00 \mathrm{~mm}(95 \% \mathrm{Cl}$ $0.62-1.38, \mathrm{H}_{\mathrm{g}}=0.74, \mathrm{CL}=70 \%$ ) than girls.

For normalised value of the $\mathrm{OE}$, there was no significant effect of sport practice and interaction ( $p>0.05$ ), but a significant effect was found for gender $(p<0.05)$ (Fig. 3B). A subsequent ANOVA with Tukey post hoc analysis showed that boys have significantly thicker muscle by $0.01 \mathrm{~mm} / \mathrm{kg}^{0.88}(95 \% \mathrm{Cl} 0.004-0.02$, $\left.\mathrm{H}_{\mathrm{g}}=0.33, \mathrm{CL}=59 \%\right)$ than girls.

\section{Oblique internus}

For an actual value of the Ol, a significant main effect of sport practice, gender, and interaction was demonstrated $(p<0.05)$. A subsequent ANOVA with Tukey post hoc analysis showed that athlete boys have significantly thicker muscle by $1.16 \mathrm{~mm}(95 \% \mathrm{Cl}$ $0.24-2.06, \mathrm{H}_{\mathrm{g}}=0.66, \mathrm{CL}=68 \%$ ) than non-athlete boys, and at least $2.01 \mathrm{~mm}\left(95 \% \mathrm{Cl} 1.04-2.97, \mathrm{H}_{\mathrm{g}}=1.40\right.$, $\mathrm{CL}=84 \%)$ thicker than athlete and non-athlete girls. Additionally, compared to non-athlete boys, non-athlete girls have thinner muscle by $1.02(95 \% \mathrm{Cl} 0.16-1.89$,
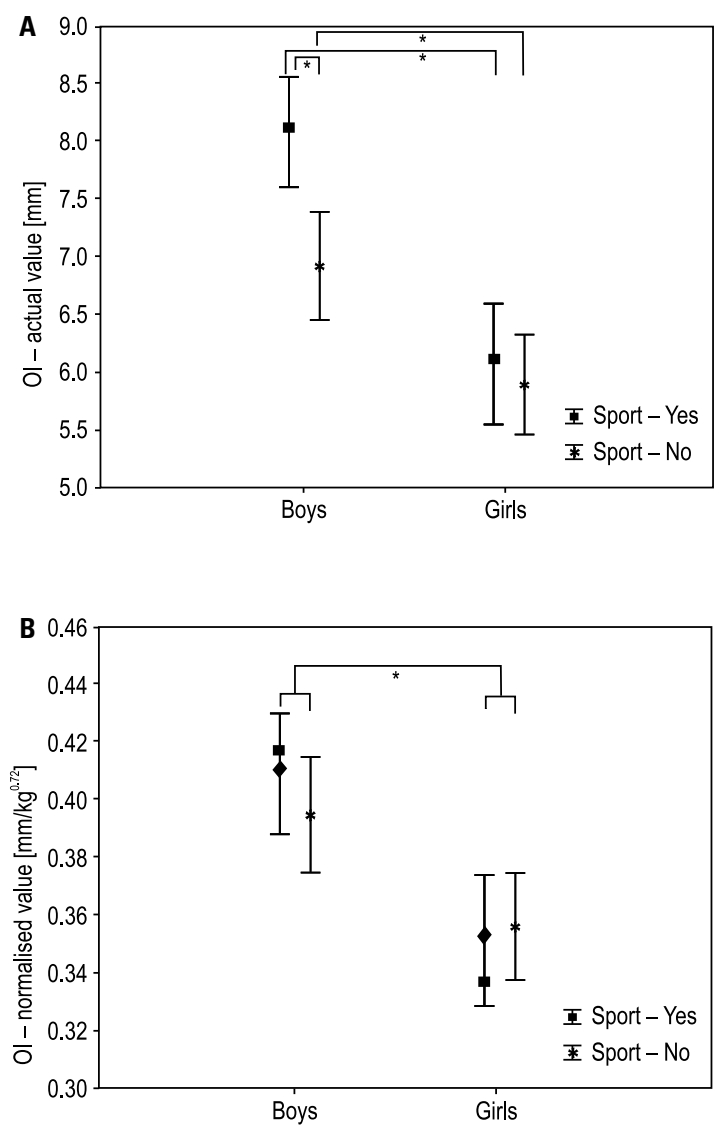

Figure 4. Oblique internus muscle (OI) before (A) and after (B) normalisation in examined groups; ${ }^{*} p<0.001$.

$H_{g}=0.57, C L=66 \%$ ) (Fig. $4 A$ ). With regard to the main effect of sport practice, the athlete group had thicker Ol muscle by $0.79 \mathrm{~mm}(95 \% \mathrm{Cl} 0.31-1.27$, $H_{g}=0.44, C L=62 \%$ ) than the non-active group.

For normalised value of the OI (Fig. 4B), there was no significant effect of sport practice and interaction $(p>0.05)$, but a significant effect was found for gender $(p<0.05)$. A subsequent ANOVA with Tukey post hoc analysis showed that boys have significantly thicker muscle by $0.04 \mathrm{~mm} / \mathrm{kg}^{0.72}$ (95\% Cl 0.03-0.07, $\mathrm{H}_{\mathrm{g}}=0.71, \mathrm{CL}=69 \%$ ) than girls.

\section{Age effect}

In non-athletes, there was a significant correlation $(p<0.01)$ between participants' age and the actual value of the OE (boys: $r=0.65$; girls: $r=0.60$ ). In athletes, there was no significant correlation between age and the actual value of the $\mathrm{OE}$ $(p>0.06)$. For normalised value of the $O E$, there was no significant correlation in either athlete (boys: $r=-0.26, p=0.08$; girls: $r=0.003, p=0.98$ ) or 
non-athlete (boys: $r=0.09, p=0.53$; girls: $r=0.13$, $p=0.34$ ) adolescents.

In non-athletes, there was a significant correlation $(p<0.01)$ between participants' age and the actual value of the OI (boys: $r=0.68$; girls: $r=0.55$ ). In athletes, there was a statistical threshold between participants' age and the actual value of the $\mathrm{OI}$ in both boys $(r=0.30, p=0.05)$ and girls $(r=0.30$, $p=0.07$ ). For normalised OI value and participants' age, there were no significant correlations (boys: $r=0.14, p=0.15 ;$ girls: $r=0.04, p=0.67$ ).

\section{DISCUSSION}

The main aim of the study was to show that omitting body mass in the analysis can lead to improper interpretation of the OAM thickness differences in examined groups. As in a prior publication [10], the gender, age, and physical activity effect on $\mathrm{Ol}$ and $\mathrm{OE}$ muscle thickness in both conditions (without body mass and with body mass) was analysed.

\section{Gender effect}

Without the inclusion of body mass, the study's results indicated that athlete and non-athlete boys have thicker $\mathrm{OI}$ and $\mathrm{OE}$ muscles than athlete and non-athlete girls with large and medium effect size, respectively. Thus, there was at least $70 \%$ probability that for randomly selected pairs of individuals (male vs. female), the OAM are thicker in male adolescents. This difference may be easily explained by the body mass, because males have higher body mass value than females. However, for allometric-scaled OE and Ol measurements, there were still significant differences between genders with small and medium effect size, respectively. This means that in the adolescent population, regardless of body mass value, there was $59 \%$ (for OE) and $69 \%$ (for OI) probability that for randomly selected pairs of individuals (male vs. female), the OAM are thicker in male adolescents.

In adult and adolescent populations, it was also identified that males have thicker OI [17-19, 22, 23] and $\mathrm{OE}[17,19,22]$ muscle than females, but the body mass was also higher in males [18, 19, 22, 23]. Thus, this cross-gender difference in OAM may again be easily explained by the body mass, but Rankin et al. [22] confirmed that after normalisation of muscle morphology for body mass, the OE and OI thicknesses were still higher in males than females, which supports the results from this study. This implies that any crossgender differences in OE and OI thickness in a healthy population are caused by gender effect, not by body mass. Only Mannion et al.'s [18] study results for $\mathrm{OE}$ thickness did not correspond with other studies' [19, 22] and this study's results, because they did not find a significant gender difference in OE thickness despite the higher body mass value in male participants.

\section{Sport practice effect}

In this study, physically active adolescents had thicker OE and OI muscle than non-active ones, with medium and small effect size, respectively. Thus, there was at least $62 \%$ probability that for randomly selected pairs of individuals (active vs. non-active), the OAM are thicker in active adolescents. This group difference can also be explained by body mass, because physically active participants were significantly heavier than non-active ones, and after body mass normalisation for the $\mathrm{OE}$ and $\mathrm{OI}$, there were no differences in allometric-scaled $\mathrm{OE}$ and $\mathrm{OI}$ thicknesses between the two examined groups. In Linek's [9] study, adolescent volleyball players had thicker OI muscle compared to the non-active control group, but in the sport group the mean body mass was higher by $6.7 \mathrm{~kg}$ than that of the controls. Hence, there is an easy explanation for why volleyball players had higher OI thickness. In another study, physically active females had similar OE and $\mathrm{OI}$ muscle thicknesses and body mass values as non-active ones [13]. Thus, it was concluded that regular physical activity does not have any effect on OAM morphology [13], and the conclusion is supported by the present study results. However, Sitilertpisan et al. [26] showed that weightlifters have significantly thicker OI muscle (but not OE) than a well-matched (in terms of body mass) control group.

The increased thickness (expected hypertrophy) of the $\mathrm{Ol}$ is a possible result of physical activity (intense training) and competition. In professional cricket players, the mean value of the Ol thickness $(16 \mathrm{~mm})$ is higher by around $8.0 \mathrm{~mm}$ than in the general adult population $(8 \mathrm{~mm})[6,18,19,22]$, and this difference is not accounted for by body mass. Thus, in Linek's [9] work, the higher Ol thickness in volleyball players may be real, but whether it is due to the lack of body mass normalisation is questionable. Taking into account that cricket players had $16 \mathrm{~mm}$ Ol thickness and weightlifters only $10 \mathrm{~mm}$, it suggests that hypertrophy changes of the abdominal muscles could have been caused by the repetition of some movement patterns in different sports, but the sport effect is probably different for each sport. Nonetheless, this 
clearly indicates that body mass normalisation for OAM is warranted and needed to obtain proper and true results in further studies of the effect of sport and/or physical activity on OE and OI thickness.

\section{Age effect}

To date, the age effect on OAM has not been examined in an adolescent population. The results of this paper suggest that there is a significant positive correlation between the actual value of the OAM and age, whereas for allometric-scaled $\mathrm{OI}$ and $\mathrm{OE}$ thickness, the correlation with age was low and insignificant. The impact of age on the OAM in the adult population has been examined in prior studies [7, 18, 22]. Rankin et al. [22] found a negative, inconsistent correlation between genders and a very low correlation $(r<-0.35)$ between age and OAM thicknesses in healthy participants aged between 20 and 72; Mannion et al. [18] examined a similar population and also found a low, negative correlation between age and OI thickness (standardised beta $=$ -0.264). Probably due to different statistical procedures in both mentioned studies, Mannion et al. [18] found no correlation between age and OE muscle. In turn, Ikezoe et al. (2012) [7] found significantly higher OAM thicknesses in young (mean age 20.0) subjects compared to independent elderly participants (mean age 85.7), but they did not consider body mass as a possible source of the disproportion between examined populations.

Taking into account that in Ikezoe et al.'s [7] study the young participants were heavier by more than $10 \mathrm{~kg}$ compared to the independent elderly group, it may be expected that after body mass normalisation the disproportion in OAM will be less visible or even disappear. In the examined adolescent population, the presence of correlations between age and OAM thicknesses was probably caused by body mass, which physiologically increased between the ages of 10 and 17 years [2]. Thus, in a healthy adolescent population there is no correlation between normalised $\mathrm{OE}$ and $\mathrm{OI}$ thicknesses and participants' age. In other words, in the second decade of life (and probably later, but Ikezoe et al.'s [7] study needs recalculation), there are no changes independent of body mass in OE and OI thicknesses at different ages.

\section{Limitations of the study}

The study has some limitations: a) in the study some participants may have scoliosis (reflected by ATR value) what can influence $\mathrm{OE}$ and $\mathrm{OI}$ morphology as in prior study (Linek et al., 2016); b) arm and leg domi- nance was not considered at all but in some sports abdominal muscles hypertrophy on one body side is observed; c) some types of sport were included as a one group in the study but it is possible that separate assessment for each sport nay bring some physical activity effect on abdominal muscles; d) some athletes involved in unilateral sports may have significant sideto-side differences in $\mathrm{OE}$ and $\mathrm{OI}$ morphology and this may led to different conclusions.

\section{CONCLUSIONS}

Analysis of OAM without body mass normalisation can lead to incorrect interpretations of study results. Thus, it is recommended that in future studies allometric-scaled $\mathrm{OE}$ and $\mathrm{OI}$ thicknesses should be analysed, rather than the actual values. The results of the present study suggest that, in the adolescent population: a) there is no effect of age and physical activity on allometric-scaled OAM thicknesses, and b) boys have thicker allometric-scaled OAM than females.

\section{REFERENCES}

1. Cumming G. Understanding the New Statistics: Effect Sizes, Confidence Intervals, and Meta-Analysis. Routledge, New York: NY 2012.

2. DiMeglio A, Dimeglio A, Canavese F, et al. Growth and adolescent idiopathic scoliosis: when and how much? J Pediatr Orthop. 2011; 31(1 Suppl): S28-S36, doi: 10.1097/ BPO.0b013e318202c25d, indexed in Pubmed: 21173616.

3. Ehsani F, Arab AM, Jaberzadeh S, et al. Ultrasound measurement of deep and superficial abdominal muscles thickness during standing postural tasks in participants with and without chronic low back pain. Man Ther. 2016; 23: 98-105, doi: 10.1016/j.math.2016.01.003, indexed in Pubmed: 26842677.

4. Fong DY, Lee CF, Cheung KM, et al. A meta-analysis of the clinical effectiveness of school scoliosis screening. Spine (Phila Pa 1976). 2010; 35(10): 1061-1071, doi: 10.1097/ BRS.0b013e3181 bcc835, indexed in Pubmed: 20393399.

5. Gray J, Aginsky KD, Derman W, et al. Symmetry, not asymmetry, of abdominal muscle morphology is associated with low back pain in cricket fast bowlers. J Sci Med Sport. 2016; 19(3): 222-226, doi: 10.1016/j.jsams.2015.04.009, indexed in Pubmed: 26059231.

6. Hides J, Wilson S, Stanton W, et al. An MRI investigation into the function of the transversus abdominis muscle during "drawing-in" of the abdominal wall. Spine (Phila Pa 1976). 2006; 31(6): E175-E178, doi: 10.1097/01. brs.0000202740.86338.df, indexed in Pubmed: 16540858.

7. Ikezoe T, Mori N, Nakamura M, et al. Effects of age and inactivity due to prolonged bed rest on atrophy of trunk muscles. Eur J Appl Physiol. 2012; 112(1): 43-48, doi: 10.1007/ s00421-011-1952-x, indexed in Pubmed: 21472438.

8. Jaric S, Mirkov D, Markovic G. Normalizing physical performance tests for body size: a proposal for standardization. J Strength Cond Res. 2005; 19(2): 467-474, doi: 10.1519/ R-15064.1, indexed in Pubmed: 15903392. 
9. Linek P. Assessment of the deep abdominal muscles at rest and during the abdominal drawing-in maneuver in adolescents practicing volleyball: a case control study. Isokinet Exerc Sci. 2015; 23(4): 215-220, doi: 10.3233/ lies-150596.

10. Linek P. The importance of body mass normalisation for ultrasound measurement of the transversus abdominis muscle: The effect of age, gender and sport practice. Musculoskelet Sci Pract. 2017; 28: 65-70, doi: 10.1016/j. msksp.2017.01.014, indexed in Pubmed: 28189038.

11. Linek $P$, Saulicz E, Kuszewski $M$, et al. Ultrasound Assessment of the Abdominal Muscles at Rest and During the ASLR Test Among Adolescents With Scoliosis. Clin Spine Surg. 2017; 30(4): 181-186, doi: 10.1097/ BSD. 0000000000000055 , indexed in Pubmed: 28437332.

12. Linek P, Saulicz E, Wolny $T$, et al. Reliability of B-mode sonography of the abdominal muscles in healthy adolescents in different body positions. J Ultrasound Med. 2014; 33(6): 1049-1056, doi: 10.7863/ultra.33.6.1049, indexed in Pubmed: 24866612.

13. Linek P, Saulicz E, Wolny $T$, et al. Assessment of the abdominal muscles at rest and during abdominal drawing-in manoeuvre in adolescent physically active girls: a case control study. J Sport Heal Sci. 2017; 6(1): 118-124, doi: 10.1016/j.jshs.2015.10.002.

14. Linek P, Saulicz E, Wolny $T$, et al. Body mass normalization for ultrasound measurements of adolescent lateral abdominal muscle thickness. J Ultrasound Med. 2017; 36(4): 775-782, doi: 10.7863/ultra.16.03086, indexed in Pubmed: 27943381.

15. Linek $P$, Saulicz $E$, Wolny $T$, et al. Intra-rater reliability of B-mode ultrasound imaging of the abdominal muscles in healthy adolescents during the active straight leg raise test. PM R. 2015; 7(1): 53-59, doi: 10.1016/j. pmrj.2014.07.007, indexed in Pubmed: 25088316.

16. Linek P, Saulicz E, Wolny T, et al. Ultrasound evaluation of the symmetry of abdominal muscles in mild adolescent idiopathic scoliosis. J Phys Ther Sci. 2015; 27(2): 465-468, doi: 10.1589/jpts.27.465, indexed in Pubmed: 25729192.

17. Linek P, Saulicz E, Wolny T, et al. Lateral abdominal muscle size at rest and during abdominal drawing-in manoeuvre in healthy adolescents. Man Ther. 2015; 20(1): 117-123, doi: 10.1016/j.math.2014.07.009, indexed in Pubmed: 25088309.

18. Mannion AF, Pulkovski N, Toma V, et al. Abdominal muscle size and symmetry at rest and during abdominal hollowing exercises in healthy control subjects. J Anat. 2008; 213(2): 173-182, doi: 10.1111/j.1469-7580.2008.00946.x.
19. Manshadi FD, Parnianpour M, Sarrafzadeh J, et al. Abdominal hollowing and lateral abdominal wall muscles' activity in both healthy men \& women: An ultrasonic assessment in supine and standing positions. J Bodyw Mov Ther. 2011; 15(1): 108-113, doi: 10.1016/j.jbmt.2009.10.004, indexed in Pubmed: 21147426.

20. McGraw K, Wong SP. A common language effect size statistic. Psychological Bulletin. 1992; 111(2): 361-365, doi: 10.1037/0033-2909.111.2.361.

21. Nuzzo JL, Mayer JM. Body mass normalisation for ultrasound measurements of lumbar multifidus and abdominal muscle size. Man Ther. 2013; 18(3): 237-242, doi: 10.1016/j. math.2012.10.011, indexed in Pubmed: 23149061.

22. Rankin G, Stokes M, Newham DJ. Abdominal muscle size and symmetry in normal subjects. Muscle Nerve. 2006; 34(3): 320-326, doi: 10.1002/mus.20589, indexed in Pubmed: 16775833.

23. Rho M, Spitznagle T, Van Dillen L, et al. Gender differences on ultrasound imaging of lateral abdominal muscle thickness in asymptomatic adults: a pilot study. PM R. 2013; 5(5): 374-380, doi: 10.1016/j.pmrj.2013.03.003, indexed in Pubmed: 23474210.

24. Rostami M, Ansari M, Noormohammadpour $P$, et al. Ultrasound assessment of trunk muscles and back flexibility, strength and endurance in off-road cyclists with and without low back pain. J Back Musculoskelet Rehabil. 2015; 28(4): 635-644, doi: 10.3233/BMR-140559, indexed in Pubmed: 25391328.

25. Rostami M, Noormohammadpour P, Mansournia MA, et al. Comparison of the thickness of lateral abdominal muscles between pregnant women with and without low back pain. PM R. 2015; 7(5): 474-478, doi: 10.1016/j. pmrj.2014.10.014, indexed in Pubmed: 25459655.

26. Sitilertpisan P, Pirunsan U, Puangmali A, et al. Comparison of lateral abdominal muscle thickness between weightlifters and matched controls. Phys Ther Sport. 2011; 12(4): 171-174, doi: 10.1016/j.ptsp.2011.02.002, indexed in Pubmed: 22085710.

27. Springer BA, Mielcarek BJ, Nesfield TK, et al. Relationships among lateral abdominal muscles, gender, body mass index, and hand dominance. J Orthop Sports Phys Ther. 2006; 36(5): 289-297, doi: 10.2519/jospt.2006.2217, indexed in Pubmed: 16715829.

28. Wilson A, Hides JA, Blizzard L, et al. Measuring ultrasound images of abdominal and lumbar multifidus muscles in older adults: A reliability study. Man Ther. 2016; 23: 114-119, doi: 10.1016/j.math.2016.01.004, indexed in Pubmed: 26832788. 